\title{
Velocidade do Som no Ar: Um Experimento Caseiro com Microcomputador e Balde D’água
}

(Speed of the sound in air: a homemade experiment with microcomputer and pail of water)

\author{
Wilton Pereira da Silva, Cleide M. D. P. S. e Silva, Tarso V. Fereira* \\ Jerônimo S. Rocha*, Diogo D. P. S. e Silva* e Cleiton D. P. S. e Silva ${ }^{\dagger}$ \\ Departamento de Física, Universidade Federal de Campina Grande \\ wiltonps@uol.com.br
}

Recebido em 9 de dezembro, 2002. Aceito em 18 de fevereiro, 2003.

\begin{abstract}
Este artigo propõe um experimento caseiro para a determinação da velocidade do som no ar. Para tal, foi desenvolvido um programa de computador para a geração de áudio em frequiências estabelecidas pelo usuário, e também para a apresentação dos roteiros experimentais (ensino médio e superior). O experimento proposto foi realizado por diversos alunos, em suas próprias casas, e os resultados obtidos concordam com os valores esperados.
\end{abstract}

This paper proposes a homemade experiment for the determination of the speed of the sound in air. For that, a computer program was developed for the audio generation in frequencies provided by the user, and also for the presentation of the experimental manuals (high school and college). The proposed experiment was accomplished by several students, at home and the obtained results agree with the expected values.

\section{Introdução}

Há muitos séculos já se estuda a natureza do som e, em particular, a sua forma de propagação. Existem inúmeras montagens experimentais com o objetivo de determinar a velocidade das ondas sonoras em vários meios, inclusive no ar.

A maioria dos livros-texto de Física apresenta formas simples para a determinação experimental da velocidade do som. Dentre essas formas pode-se destacar a montagem mostrada na Fig. 1 (veja, por exemplo, as referências [1], [2] e [3]).

Essa montagem consiste de um tubo flexível em U, parcialmente preenchido com água, e um diapasão que vibra com determinada freqüência, próximo do ramo do tubo que contém uma coluna de ar na parte superior. A variação do comprimento da coluna de ar é obtida pela modificação na altura do outro ramo do tubo em U, que contém um reservatório de água. Assim, através do som produzido pela vibração do diapasão (ou de um gerador de áudio) numa determinada freqüência, é possível variar o comprimento da coluna de ar até um valor tal que ocorra a ressonância, devido à reflexão das ondas na superfície líquida (interface arágua). Observando que tal coluna de ar constitui um caso clássico de tubo com uma extremidade aberta e outra fechada, pode-se determinar a velocidade do som no ar através de cálculos simples.

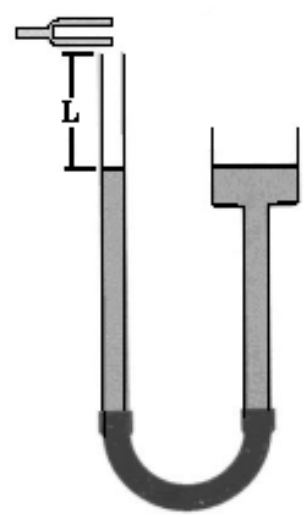

Figura 1. Esquema para a determinação da velocidade do som no ar.

Nos últimos anos, com a popularização dos microcomputadores, têm surgido várias propostas de montagens para a determinação da velocidade do som (veja, por exemplo, [4] e [5]). Tais montagens envolvem não só o micro, em si, mas também circuitos eletrônicos com microfone, alto-falante e outros componentes.

\footnotetext{
** Bolsistas do projeto "Aperfeiçoamento de Professores no Ensino de Física", UFCG

${ }^{\dagger * *}$ Mestrado em Engenharia Eletrônica, ITA
} 
Qualquer que seja o tipo de montagem para a determinação da velocidade do som no ar, sente-se a necessidade do "ambiente do laboratório". No caso clássico, por mais simples que seja a montagem, deve haver a disponibilidade de tubos, mangueira, reservatório de água (funil), cola e um conjunto de diapasões ou um gerador de áudio. Apesar da montagem não requerer mão de obra especializada, é possível que muitos laboratórios de ensino de muitas universidades (e de escolas de nível médio) não disponham de alguns dos equipamentos necessários para a realização do experimento. Já a determinação da velocidade do som com a utilização de um micro associado a circuitos eletrônicos requer, além de uma mão de obra mais especializada para as montagens, de alguns equipamentos que também podem não estar disponíveis.

O propósito deste artigo é aliar uma das formas clássicas de determinação da velocidade do som no ar, que é a detecção de ressonâncias em um tubo, com um programa de computador que dispensa a utilização de um conjunto de diapasões, e também a parafernália eletrônica. Como conseqüência deste propósito, supõe-se que o interessado possa realizar o experimento em sua própria casa sem, contudo, renunciar ao necessário rigor em seus resultados.

\section{O experimento}

A proposta experimental ressalta três requisitos básicos, a seguir detalhados.

\section{II.1 A montagem experimental}

A montagem experimental é mostrada na Fig. 2, e consiste em um balde com água, no qual é introduzido um cano de PVC. Dessa forma, o comprimento da coluna de ar pode ser variado de forma simples, o que possibilita detectar facilmente pontos de ressonância.

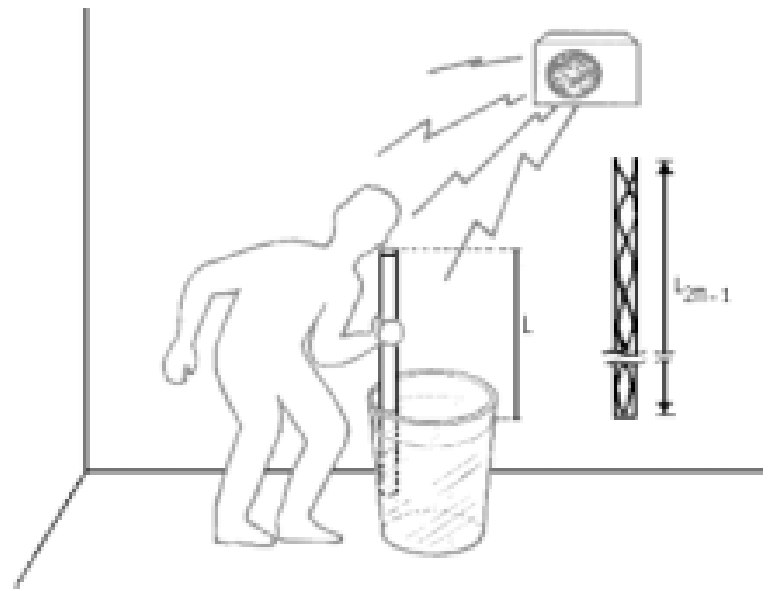

Figura 2. Montagem experimental proposta. O áudio é gerado por um programa de computador.

Essa proposta experimental elimina vários inconvenientes da montagem clássica que envolve tubos, mangueira flexível e reservatório de água, conectados conforme a Fig. 1.

\section{II.2 A geração de áudio}

Para substituir o conjunto de diapasões (ou o equipamento eletrônico gerador de áudio), pensou-se, inicialmente, na gravação de uma fita cassete. Mas obviamente a distribuição desta fita pelo país afora não seria muito prática devido, primeiro, à necessidade do pedido formal desta fita por parte do interessado e, segundo, devido ao inconveniente da remessa dessa fita via correio, o que teria um certo custo, além da natural demora no transporte. A alternativa para eliminar estes inconvenientes foi o desenvolvimento de um programa de computador. Naturalmente, o programa não elimina a possibilidade da gravação de uma fita cassete por parte de interessados, e sim facilita: um professor que tenha feito o download de tal programa pode gravar as freqüências e repassá-las àqueles alunos que não dispõem de um micro em suas casas. O software desenvolvido foi denominado "VSOM" [6] e dispõe de duas opções de geração de áudio, conforme mostra a Fig. 3.

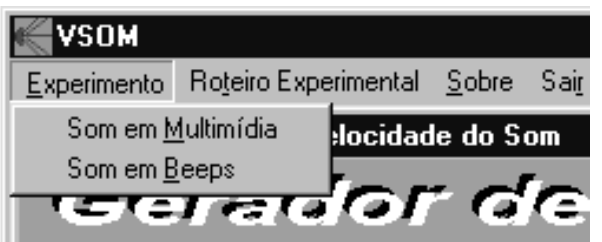

Figura 3. Opções de geração de áudio do programa desenvolvido para o experimento.

Uma das opções disponíveis pode ser útil em micros que não possuem kit multimídia: os sons podem ser gerados por "beeps", na freqüência indicada pelo usuário, como pode ser visto na caixa de diálogo mostrada na Fig. 4.

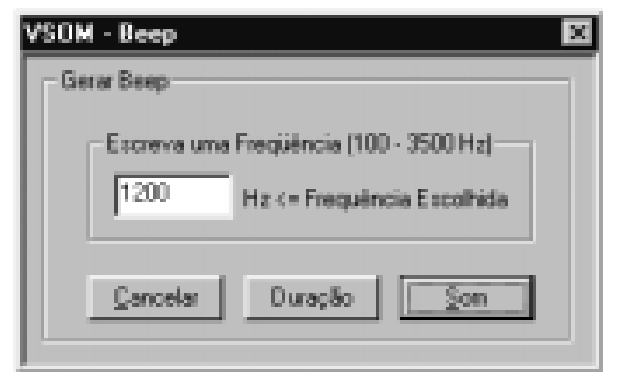

Figura 4. Caixa de diálogo do VSOM para geração de áudio (beeps) na frequiência estipulada pelo usuário.

Como em alguns micros a potência sonora de beeps não é satisfatória para a realização do experimento, outra alternativa foi a de disponibilizar um conjunto de 8 arquivos com extensão "wav", gerados com a utilização do software TTG [7]. Os arquivos produzem áudios nas freqüências de 1200 , 1600, 2000, 2400, 2800, 3200, 3600 e 4000 Hz. Tais arquivos são executados a partir da caixa de diálogo mostrada na Fig. 5. 


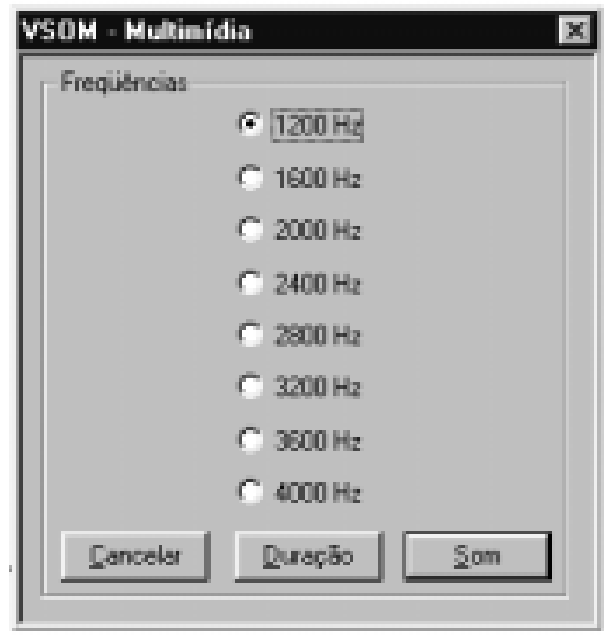

Figura 5. Caixa de diálogo do VSOM para a geração de áudio (multimídia) na frequiência selecionada pelo usuário.

\section{II.3 Roteiros experimentais}

A forma de realização da experiência é detalhada através de roteiros experimentais disponíveis no menu "Roteiro Experimental", que pode ser visto na Fig. 6.

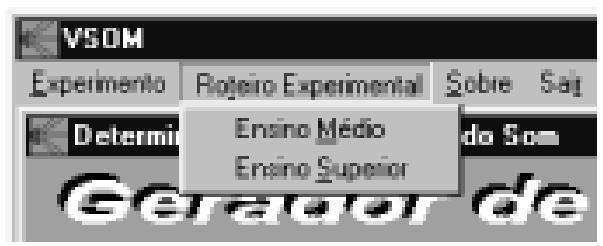

Figura 6. Roteiros experimentais disponíveis no VSOM.

Como os arquivos com os roteiros são de extensão hlp (arquivos de ajuda, tipo "leitura somente"), há a possiblidade de impressão de tais roteiros, se o usuário julgar adequado. Em anexo, é apresentado o roteiro para a realização do experimento, preparado para o ensino superior. Neste roteiro é indicada a forma de realização do experimento e é dada uma sugestão para a elaboração do relatório. Na parte dedicada à elaboração do relatório, o aluno é provocado a refletir sobre a dedução de uma expressão que possibilita a determinação da velocidade do som no ar, a partir dos dados experimentais. Além disso, o aluno é provocado, também, a deduzir uma expressão para velocidade do som em qualquer meio, seguido de uma particularização dessa expressão para o ar.

\section{Fundamentação teórica}

Como a teoria envolvendo a propagação do som em um meio é bem conhecida, serão apresentadas aqui, apenas as expressões úteis aos objetivos do experimento, sem maiores considerações. Detalhes adicionais podem ser obtidos no roteiro experimental, apresentado anexo.

\section{III.1 Comprimento do tubo de ar na res- sonância}

Conforme a descrição da montagem, o experimento consiste de um tubo com uma extremidade aberta e outra fechada. Para tubos deste tipo, o comprimento $L$ da coluna de ar relativo à ressonância é dado por

$$
L_{2 n-1}=\frac{(2 n-1)}{4} \lambda \quad \text { para } n=1,2,3, \ldots
$$

em que $\lambda$ é o comprimento da onda sonora. Assim, a diferença no comprimento do tubo para duas ressonâncias consecutivas é dada por

$$
\Delta L=\frac{V_{\text {som }}}{2} \frac{1}{f}
$$

em que $f$ é a freqüência.

\section{III.2 Velocidade de propagação do som num dado meio}

No roteiro experimental no anexo é proposto aos alunos um estudo visando demonstrar que a velocidade do som em um meio material é dada por

$$
V=\sqrt{\frac{Y}{\rho}}
$$

sendo $Y$ o módulo de Young do meio e $\rho$ a sua densidade. Para o caso do ar, em que as compressões e expansões produzidas pela propagação podem ser consideradas adiabáticas, a Eq. (3) reduz-se a

$$
V=\sqrt{\frac{\gamma R T}{M}}
$$

em que $\gamma=\mathrm{C}_{P} / \mathrm{C}_{V}$ vale 1,40 , R é a constante universal dos gases ideais (igual a $8,31 \mathrm{~J} / \mathrm{mol} . \mathrm{K}$ ) e $\mathrm{M}$ é a massa molecular do ar, e vale $29,0 \times 10^{-3} \mathrm{~kg} / \mathrm{mol}$.

\section{Realização do experimento}

O experimento proposto foi realizado numa temperatura de $23{ }^{0} \mathrm{C}$, para as freqüências disponíveis na opção "multimídia”. Os dados obtidos são apresentados na Tabela I.

\begin{tabular}{|c|c|c|c|c|c|c|c|c|}
\hline & 1 & 2 & 3 & 4 & 5 & 6 & 7 & 8 \\
\hline$f(\mathrm{~Hz})$ & 1200 & 1600 & 2000 & 2400 & 2800 & 3200 & 3600 & 4000 \\
\hline$\Delta \mathrm{L}(\mathrm{m})$ & 0,144 & 0,110 & 0,083 & 0,070 & 0,060 & 0,053 & 0,050 & 0,043 \\
\hline
\end{tabular}

Tabela I - Freqüências e as correpondentes distâncias entre duas ressonâncias consecutivas 
A representação gráfica entre a distância de duas ressonâncias consecutivas $\Delta L$ versus a freqüência $f$ indica que a relação funcional entre essas grandezas é dada por uma hipérbole, conforme mostra a Fig. 7, prevista teoricamente pela Eq. 2.

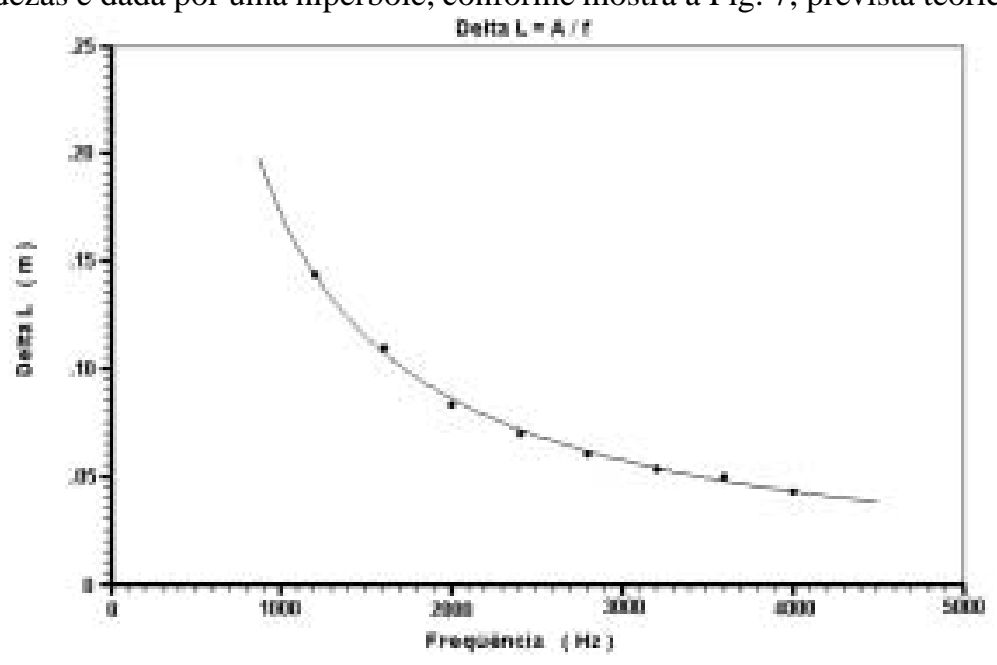

Figura 7. Distância entre duas ressonâncias consecutivas versus a freqüência.

Assim, usando o software de ajuste de curvas denominado LabFit [8] para ajustar a função

$$
\Delta L=A \frac{1}{f}
$$

aos pontos experimentais, obtém-se $A=(171,9 \pm 1,4) \mathrm{m} / \mathrm{s}$. Através da comparação da Eq. 5 com a Eq. 2, tem-se que

$$
V_{\text {exp }}=2 A,
$$

o que resulta no seguinte valor experimental para a velocidade do som no ar: $\mathrm{V}_{\text {exp }}=(343,8 \pm 2,8) \mathrm{m} / \mathrm{s}$. Por outro lado, para a temperatura em que o experimento foi realizado, $\mathrm{T}=296 \mathrm{~K}$, e lembrando que $\mathrm{M}=29,0 \times 10^{-3} \mathrm{~kg} / \mathrm{mol}, \mathrm{R}=$ $8,31 \mathrm{~J} / \mathrm{mol} . \mathrm{K}$ e $\gamma=1,40$, obtém-se, pela Eq. $4: \mathrm{V}_{t e o}=345$ $\mathrm{m} / \mathrm{s}$.

\section{Conclusões}

Supondo que o valor teórico previsto seja o valor correto para a velocidade do som no ar, o resultado experimental obtido contém um erro de $0,3 \%$. Então, pode-se concluir que tal resultado é bastante satisfatório, levando-se em conta os objetivos educacionais da proposta apresentada.

O experimento foi realizado, a pedido de seus criadores, por vários alunos, em suas próprias casas. Os resultados obtidos, com erros inferiores a 5\%, são semelhantes aos que foram apresentados aqui. Isto reforça o propósito do experimento, que é o de disponibilizar uma forma prática e simples para a determinação da velocidade do som no ar. Por "simples", deve-se entender a realização do experimento sem grandes dificuldades, seja no acesso aos materiais e roteiro, ou ainda com relação à montagem e obtenção dos da- dos ou mesmo quanto ao tratamento de tais dados sem, contudo, abrir mão de bons resultados.

Por último, há que se ressaltar que o roteiro experimental proposto possibilita uma revisão ampla sobre a propagação de ondas em meios mecânicos o que, em si, já é um subproduto positivo dos objetivos delineados, embora não tenha sido enfatizado na proposta experimental.

\section{Referências}

[1] Tipler, Paul A. Física, V.2, LTC - Livros Técnicos e Científicos Editora S. A., Rio de Janeiro, $3^{a}$ Edição (1995), pag. 152

[2] Halliday, David e Resnick, Robert, Física, Vol. 1-2, LTC Livros Técnicos e Científicos Editora S. A., Rio de Janeiro (1977), pag. 569

[3] Silva, Wilton P. e Silva, Cleide M. D. P. S., Mecânica Experimental para Físicos e Engenheiros, Ed. Universitária, João Pessoa (2000), pag. 279

[4] Barbeta, Vagner B. e Marzzulli, Cláudia R., Rev. Bras. Ens. Fís., 22 (4), (2000) pag. 447-455

[5] Worland, R. S. e Wilson, D. D.,The Phys. Teacher 37, 53 (1999)

[6] Software VSOM: http://us.share.geocities.com/pextensao/Vsom.zip www.extensao.hpg.com.br

[7] Software Test Tone Generator (TTG): www.esser.u-net.com/ttg.htm

[8] Lab Fit Curve Fitting Software: www.angelfire.com/rnb/labfit 
ANEXO

\section{Roteiro para a determinação da velocidade do som no ar}

(Nível superior)

\section{Objetivos}

Determinar a velocidade do som no ar através da detecção da ressonância de ondas sonoras num tubo.

\section{Material}

Régua milimetrada, programa gerador de freqüências (áudio), microcomputador, $60 \mathrm{~cm}$ de cano de $32 \mathrm{~mm}$ (tubo de PVC) e balde d'água com altura mínima de $50 \mathrm{~cm}$.

\section{Montagem}

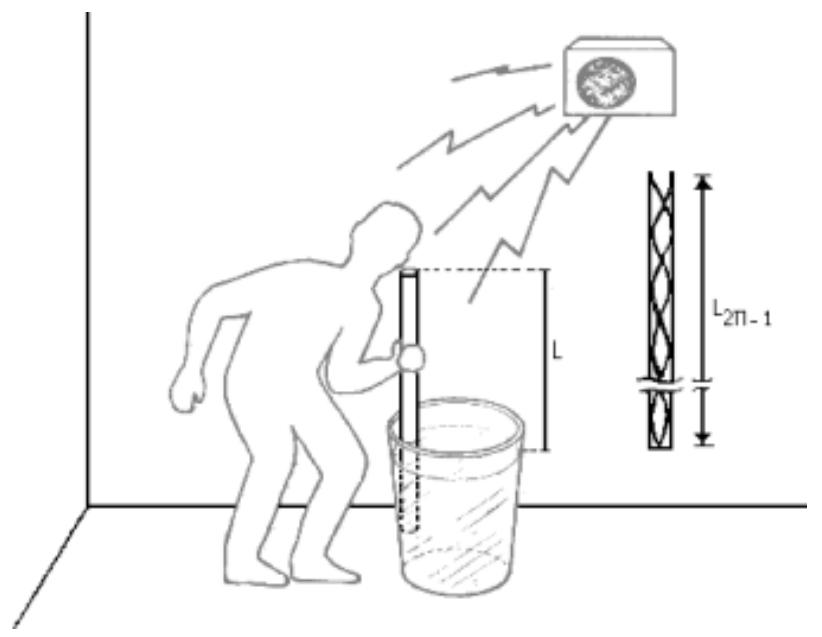

\section{Procedimentos}

1) Encha o balde de água. Avalie (ou meça, se tiver um termômetro) e anote a temperatura ambiente.
2) Execute o programa gerador de áudio para a freqüência de $1200 \mathrm{~Hz}$. Você pode optar por sons gerados por "beeps" ou "multimídia" mas, caso seu micro tenha kit multimídia, escolha essa opção no programa.

3) Para diminuir o comprimento da coluna vertical de ar no cano introduza, lentamente, uma de suas extremidades na água do balde. Ao mesmo tempo, coloque o ouvido próximo à outra extremidade, conforme mostra a figura relativa à montagem. Quando a ressonância for atingida, através da reflexão das ondas na superfície da água no interior do tubo, tem-se a impressão de que essas ondas estão sendo emitidas de dentro do tubo. Isso acontece devido ao aumento do volume do som ocorrido por causa do reforço na energia da onda sonora.

4) Através de sua percepção sonora, faça um ajuste fino na posição vertical do tubo, como quem procura a melhor sintonia de uma emissora de rádio. Para essa posição, meça e anote a distância $L_{1}$ da extremidade superior do tubo até a lâmina d'água (como o interesse será na distância entre pontos referentes a duas ressonâncias consecutivas pode-se medir, por comodidade, a distância da extremidade livre do cano até a borda do balde).

5) Repita os procedimentos 2, 3 e 4, diminuindo a coluna de ar (isto é, introduzindo o cano na água), até obter a próxima ressonância. Meça e anote a nova distância $L_{3}$ da extremidade superior do tubo até a lâmina d'água (ou até a borda do balde).

6) Repita os procedimentos 2, 3 e 4 para obter uma terceira ressonância consecutiva.

7) Repita os procedimentos anteriores para as freqüências de 1600 até $4000 \mathrm{~Hz}$.

Atenção: como será visto, o que interessa medir, de fato, é a distância entre duas ressonâncias consecutivas. Uma forma prática de obter-se diretamente a distância $\Delta L$ entre duas ressonâncias consecutivas, ao invés de $L$, é fazer marcas com lápis no cano, tomando-se como ponto de referência a borda do balde.

\section{Medidas}

Temperatura ambiente $\mathrm{t}=$ ${ }^{0} \mathrm{C}$

FREQÜÊNCIA DE $1200 \mathrm{~Hz}$

Primeira ressonância Segunda ressonância

Terceira ressonância

$$
\begin{gathered}
L_{1}= \\
L_{3}= \\
L_{5}=
\end{gathered}
$$
.$c m$ $\mathrm{cm}$ . $\mathrm{cm}$

$$
\begin{array}{cc}
\text { ou } & L_{1}=\ldots \ldots \ldots \ldots \ldots m \\
\text { ou } & L_{3}=\ldots \ldots \ldots \ldots \ldots m \\
\text { ou } & L_{5}=\ldots \ldots \ldots \ldots \ldots m
\end{array}
$$

FREQÜÊNCIA DE $1600 \mathrm{~Hz}$

Primeira ressonância Segunda ressonância Terceira ressonância
$L_{1}=$

$L_{3}=$

$L_{5}=$ cm

$\mathrm{cm}$

cm
ou
$L_{1}=$ .m
ou $L_{3}=\ldots \ldots \ldots \ldots \ldots m$
ou $\quad L_{5}=\ldots \ldots \ldots \ldots \ldots m$ 
FREQÜÊNCIA DE $2000 \mathrm{~Hz}$

$\begin{array}{llll}\text { Primeira ressonância } & L_{1}=\ldots \ldots \ldots \ldots . . c m & \text { ou } & L_{1}=\ldots \ldots \ldots \ldots \ldots m \\ \text { Segunda ressonância } & L_{3}=\ldots \ldots \ldots \ldots \ldots c m & \text { ou } & L_{3}=\ldots \ldots \ldots \ldots \ldots . m \\ \text { Terceira ressonância } & L_{5}=\ldots \ldots \ldots \ldots \ldots . m & \text { ou } & L_{5}=\ldots \ldots \ldots \ldots \ldots . m\end{array}$

FREQÜÊNCIA DE $2400 \mathrm{~Hz}$

$\begin{array}{llll}\text { Primeira ressonância } & L_{1}=\ldots \ldots \ldots \ldots \ldots . c m & \text { ou } & L_{1}=\ldots \ldots \ldots \ldots \ldots . m \\ \text { Segunda ressonância } & L_{3}=\ldots \ldots \ldots \ldots \ldots . m & \text { ou } & L_{3}=\ldots \ldots \ldots \ldots \ldots . . m \\ \text { Terceira ressonância } & L_{5}=\ldots \ldots \ldots \ldots \ldots . m & \text { ou } & L_{5}=\ldots \ldots \ldots \ldots \ldots . m\end{array}$

FREQÜÊNCIA DE $2800 \mathrm{~Hz}$

$\begin{array}{llll}\text { Primeira ressonância } & L_{1}=\ldots \ldots \ldots \ldots \ldots c m & \text { ou } & L_{1}=\ldots \ldots \ldots \ldots \ldots m \\ \text { Segunda ressonância } & L_{3}=\ldots \ldots \ldots \ldots \ldots c m & \text { ou } & L_{3}=\ldots \ldots \ldots \ldots \ldots . m \\ \text { Terceira ressonância } & L_{5}=\ldots \ldots \ldots \ldots \ldots . m & \text { ou } & L_{5}=\ldots \ldots \ldots \ldots \ldots . m\end{array}$

FREQÜÊNCIA DE $3200 \mathrm{~Hz}$

Primeira ressonância $\quad L_{1}=\ldots \ldots \ldots \ldots . . c m \quad$ ou $\quad L_{1}=\ldots \ldots \ldots \ldots \ldots . . m$

Segunda ressonância $\quad L_{3}=\ldots \ldots \ldots \ldots . . c m \quad$ ou $\quad L_{3}=\ldots \ldots \ldots \ldots \ldots . . m$

Terceira ressonância $\quad L_{5}=\ldots \ldots \ldots \ldots \ldots . c m \quad$ ou $\quad L_{5}=\ldots \ldots \ldots \ldots \ldots . m$

FREQÜÊNCIA DE $3600 \mathrm{~Hz}$

Primeira ressonância $\quad L_{1}=\ldots \ldots \ldots \ldots \ldots . c m \quad$ ou $\quad L_{1}=\ldots \ldots \ldots \ldots \ldots . . m$

Segunda ressonância $\quad L_{3}=\ldots \ldots \ldots \ldots . . \mathrm{cm} \quad$ ou $\quad L_{3}=\ldots \ldots \ldots \ldots \ldots . . . m$

Terceira ressonância $\quad L_{5}=\ldots \ldots \ldots \ldots \ldots . c m \quad$ ou $\quad L_{5}=\ldots \ldots \ldots \ldots \ldots . . m$

FREQÜÊNCIA DE $4000 \mathrm{~Hz}$

$\begin{array}{llll}\text { Primeira ressonância } & L_{1}=\ldots \ldots \ldots \ldots . . \mathrm{cm} & \text { ou } & L_{1}=\ldots \ldots \ldots \ldots \ldots m \\ \text { Segunda ressonância } & L_{3}=\ldots \ldots \ldots \ldots \ldots \mathrm{cm} & \text { ou } & L_{3}=\ldots \ldots \ldots \ldots \ldots m \\ \text { Terceira ressonância } & L_{5}=\ldots \ldots \ldots \ldots \ldots . m & \text { ou } & L_{5}=\ldots \ldots \ldots \ldots \ldots m\end{array}$

\section{Relatório}

Veja, a seguir, um roteiro para a elaboração do seu relatório.

\section{Introdução}

$\mathrm{Na}$ introdução fale sobre os objetivos do experimento, liste o material utilizado e esquematize a montagem experimental.

\section{Procedimentos e análises}

A) Descreva os seus procedimentos experimentais e anote os dados coletados.

\section{UM MODELO EXPERIMENTAL PARA A VELOCI- DADE DO SOM NO AR}

B) Veja que o tubo com ar têm uma extremidade fechada (superfície líquida), onde as ondas são refletidas, e uma outra aberta. Para tubos desse tipo observa-se que, na ressonância, o comprimento da coluna de ar é dado por:

$$
L_{2 n-1}=\frac{(2 n-1)}{4} \lambda \quad \text { para } n=1,2,3, \ldots
$$

onde $\lambda$ é o comprimento da onda sonora. Determine $L_{1} \mathrm{e}$ $L_{3}$ e mostre-os, com um esboço das correspondentes ondas sonoras, no tubo.

C) Com a informação dada em B, mostre que a velocidade das ondas sonoras é dada por:

$$
v=(2 \Delta L) f
$$

onde $\Delta L$ é a diferença entre os comprimentos da coluna de ar para duas ressonâncias consecutivas (e, nesse experimento, é igual à diferença entre duas distâncias $L$ consecutivas) e $f$ é a frequiência da onda sonora. Então, têm-se também: 


$$
\Delta L=\frac{v}{2} \frac{1}{f}
$$

Para obter essa expressão experimentalmente, preencha a tabela a seguir, a partir de seus dados experimentais. Lembre-se de calcular cada $\Delta L$ através da média aritmética entre $\left(L_{3}-L_{1}\right)$ e $\left(L_{5}-L_{3}\right)$ :

\begin{tabular}{|l|l|l|l|l|l|l|l|l|}
\hline & 1 & 2 & 3 & 4 & 5 & 6 & 7 & 8 \\
\hline$\Delta L(m)$ & & & & & & & & \\
\hline$f(H z)$ & & & & & & & & \\
\hline
\end{tabular}

D) Ao traçar o gráfico de $\Delta L$ versus $f$, observe que a curva parece ser do tipo

$$
\Delta L=A \frac{1}{f} .
$$

Então, faça o ajuste dessa curva aos pontos experimentais e determine o parâmetro A da hipérbole. Note, comparando as expressões obtidas para $\Delta L \mathrm{em} \mathrm{C} \mathrm{e} \mathrm{em} \mathrm{D}$, que este parâmetro deve ser igual a $v / 2$. Com isso, calcule o valor da velocidade do som no ar.

\section{UM MODELO TEÓRICO PARA A VELOCIDADE DO SOM NO AR}

E) Um modelo simples para a "visualização" da propagação do som em um meio (por exemplo, numa barra longa), consiste na analogia do meio com esferas em repouso, enfileiradas ao longo de um tubo; muito próximas uma das outras. Ao impulsionarmos as esferas de uma extremidade (batendo com um pequeno martelo da esquerda para a direita), com uma pequena velocidade $\mu$, durante um intervalo de tempo $\Delta t$, todas as esferas contidas na distância $\ell=v \Delta t$ terão sido impulsionadas devido às colisões sucessivas. Observe que $v$ é a velocidade com que as colisões sucessivas foram transmitidas ao longo do tubo.

Por outro lado observe que, com o impulso, há um "encolhimento" $\Delta \ell=\mu \Delta t$ na distância $\ell$. Este encolhimento corresponde ao deslocamento das esferas da esquerda para a direita após o impulso. Portanto, a deformação devido ao impulso, dada por $D=\Delta \ell / \ell$ será

$$
D=\frac{\mu}{v}
$$

Lembrando que o impulso das esferas (contidas ao longo da distância $\ell=v \Delta t$ ) é igual à variação de sua quantidade de movimento, podemos escrever:

$$
F \Delta t=v \Delta t \rho_{\ell} \mu
$$

ou

$$
F=\rho_{\ell} v \mu
$$

em que $\rho_{\ell}$ é a densidade linear da fila de esferas.
No caso real de uma barra longa com uma seção reta de área $A$, a tensão em sua extremidade (dada por $S=F / A$ ), devido ao impulso, é:

$$
S=\frac{\rho_{\ell} v \mu}{A}
$$

$\mathrm{ou}$

$$
S=\rho v \mu,
$$

em que $\rho$ é a densidade do material, dada por $\rho=\rho_{\ell} / A$. Assim, determine uma expressão para o módulo de Young $(Y=S / D)$ da barra. Com essa expressão, mostre que a velocidade com que uma informação é transmitida na barra é dada por

$$
v=\sqrt{\frac{Y}{\rho}}
$$

Para um gás ideal submetido a processos adiabáticos (como é o caso da propagação do som nesse meio), mostre que $Y=\gamma P$, o que resulta em

$$
v=\sqrt{\frac{\gamma R T}{M}}
$$

Nessa expressão, $\gamma$ é a constante adiabática dada por $C_{P} / C_{V}, R$ é a constante universal dos gases ideais, $T$ é a temperatura absoluta do gás e $M$ é a sua massa molecular. Considere o ar como um gás ideal (com $\gamma=1,40$, $M=29,0 \times 10^{-3} \mathrm{Kg} / \mathrm{mol}$ e $R=8,31 \mathrm{~J} / \mathrm{mol} . \mathrm{K}$ ) e calcule a velocidade do som no ar.

\section{Conclusões}

F) Considere o valor para a velocidade do som obtido em E como correto, e faça uma avaliação do erro percentual cometido no valor experimental de $v$, obtido em $\mathrm{D}$.

G) Faça algumas considerações sobre a facilidade ou dificuldade encontradas tanto na aquisição do material experimental quanto na realização do experimento.

H) Reflita sobre quais valores experimentais foram mais difíceis de se medir, e o motivo. Como contornar tais dificuldades, se é que elas existiram?

I) Caso você conheça outras montagens para a determinação da velocidade do som, como as compararia com essa? Analise os aspectos de facilidade de realização, resultados experimentais, aquisição de materiais, etc.

J) No meio de duas ressonâncias consecutivas, você deve ter observado que há um "ponto de surdez". Como justificar essa observação?

K) Para o ferro, $Y=190 \mathrm{GN} / \mathrm{m}^{2}$ e $\rho=7,96 \times$ $10^{3} \mathrm{Kg} / \mathrm{m}^{3}$. Calcule a velocidade de propagação do som no ferro a partir da expressão geral obtida em E, e comparea com a velocidade de propagação no ar. Dê uma indicação dos motivos da diferença entre tais valores nesses meios distintos.

L) Faça comentários sobre o experimento e dê sugestões visando melhorar a qualidade dos resultados e a compreensão geral do tema abordado. 